\title{
UCRL-CONF-201860
}

LAWRENCE LIVERMORE NAT I ONA I LABORATORY

\section{Mixed Electromagnetic and Circuit Simulations using a Higher-Order Hybrid Formulation}

N. J. Champagne, J. D. Rockway, and

V. Jandhyala

January 15, 2004

2004 IEEE International Symposium on Antennas and Propagation and USNC/URSI National Radio Science Meeting Monterey, CA June 20-26, 2004 


\section{Disclaimer}

This document was prepared as an account of work sponsored by an agency of the United States Government. Neither the United States Government nor the University of California nor any of their employees, makes any warranty, express or implied, or assumes any legal liability or responsibility for the accuracy, completeness, or usefulness of any information, apparatus, product, or process disclosed, or represents that its use would not infringe privately owned rights. Reference herein to any specific commercial product, process, or service by trade name, trademark, manufacturer, or otherwise, does not necessarily constitute or imply its endorsement, recommendation, or favoring by the United States Government or the University of California. The views and opinions of authors expressed herein do not necessarily state or reflect those of the United States Government or the University of California, and shall not be used for advertising or product endorsement purposes.

\section{Auspices Statement}

This work was performed under the auspices of the U.S. Department of Energy by University of California, Lawrence Livermore National Laboratory under Contract W-7405-Eng-48. 


\section{Mixed Electromagnetic and Circuit Simulations using a Higher-Order Hybrid Formulation}

\author{
Nathan J. Champagne* and John D. Rockway \\ Defense Sciences Engineering Division \\ Lawrence Livermore National Laboratory \\ Livermore, CA 94550
}

\author{
Vikram Jandhyala \\ Department of Electrical Engineering \\ University of Washington \\ Seattle, WA 98195
}

\section{INTRODUCTION}

Standard surface impedance approximations are invalid at lower frequencies approaching DC since the cross sections of conductors are smaller than the skin depth. Hence, a volumetric formulation is typically used at these low frequencies for broadband simulation as necessitated in digital or ultra-wideband systems since the skin effect can be modeled explicitly. This modeling requires fine and frequency dependent volume meshing. However, an approach using higher-order elements and/or bases may alleviate these requirements. The intent of this paper is to present a tightly coupled circuit and hybrid boundary element (or integral equation)/finite element based electromagnetic simulation that has been coded in EIGER [1].

\section{HYBRID FORMULATION}

The approach used for the hybrid formulation has been presented in [2]. The body of interest is shown in Fig. 1. The surface of the body is used to create a boundary to separate the problem into interior and exterior regions. Finite elements are used in the interior volume $V$. A boundary element formulation is applied on the body surface $S$ to model the exterior. The interior volume of the body is modeled with finite elements using the electric field Helmholtz equation, represented as

$$
\nabla \times\left(\overline{\boldsymbol{\mu}}_{r}^{-1} \cdot \nabla \times \mathbf{E}\right)-k_{0}^{2} \overline{\boldsymbol{\varepsilon}}_{r} \cdot \mathbf{E}=-j \omega \mu_{0} \mathbf{J},
$$

where $\mathbf{E}$ is the electric field, $k_{0}$ is the freespace wave number, $\omega$ is the radian frequency, and $\boldsymbol{\varepsilon}_{r}$ and $\boldsymbol{\mu}_{r}$ are the relative permittivity and permeability dyads, respectively. The body exterior is modeled using boundary elements on the body surface, in this case using the electric field integral equation (EFIE), which is given by

$$
\frac{\mathbf{M}}{2}-\hat{\mathbf{n}} \times\left(j \omega \mathbf{A}+\nabla \Phi+\frac{1}{\varepsilon} \nabla \times \mathbf{F}\right)=-\hat{\mathbf{n}} \times \mathbf{E}^{i}, \quad \mathbf{r} \uparrow S,
$$

where $\hat{\mathbf{n}}$ is the normal to the surface at the observation point, $\mathbf{E}^{i}$ is the impressed electric field, and $\mathbf{A}, \Phi$, and $\mathbf{F}$ are the magnetic vector, electric scalar, and the electric vector potentials, respectively. Also, the jump discontinuity of the curl term in (2) has been removed and expressed explicitly in terms of the magnetic current $\mathbf{M}$. The fields and currents are expanded using the basis functions described in [3]. 


\section{CIRCUIT-CONNECTION ALGORITHM}

The contact-connection algorithm [4,5] is employed to couple the circuit to the electromagnetic surface. A circuit is attached to a spatially localized surface $S_{c}$ by enforcing at this contact a modified current-continuity equation, a KCL connection, and a KVL connection from the contact to the circuit node. This is shown in Fig. 2. These three conditions are fundamental to the coupled hybrid-circuit formulation. On a contact surface $S_{c}$, the continuity equation is changed to account for injecting branch current from the circuit. This current introduces an additional source term in the continuity equation and thus affects the distribution of both the electromagnetic surface currents and surface charges. Hence, the continuity equation is modified to become

$$
\nabla \cdot \mathbf{J}+j \omega \rho=\left\{\begin{array}{c}
I_{c}, \quad r \in S_{c}, \\
0, \text { otherwise, }
\end{array}\right.
$$

where $I_{c}$ is the contact current. This contact current provides a virtual extension from the distributive electromagnetic surface to the circuit node. The coupling between the electromagnetic formulation and the circuit is done by enforcing (2) at the boundary and coupling them to the circuit by including the contact current from (3). The localized circuit source attached to the contact produces an additional source or sink of charge that alters the scalar potential and the resulting electric field. Because of this additional current, the scalar potentials must be tied to the circuit node voltage $\mathrm{V}_{\mathrm{n}}$. A KVL expression sets the scalar potentials at the equipotent circuit voltage $V_{n}$. Finally, the contact current is connected to the circuit by including an addition term $I_{c}$ to the KCL based circuit equation associated at circuit node $n$.

The hybrid-circuit formulation, including the connecting KCL and equipotential KVL equations, may be summarized as the following block-matrix equation:

$$
\left[\begin{array}{ccc}
F E M & C_{A} & 0 \\
C_{B} & B E M & X \\
0 & -X^{T} & M N A
\end{array}\right]\left[\begin{array}{l}
V_{E M} \\
I_{E M} \\
I_{c k t}
\end{array}\right]=0,
$$

where the FEM block matrix represents the finite elements, $B E M$ represents the boundary elements and contacts, and the $C$ blocks represent the cross-coupling between the finite elements and boundary elements. The $X$ and $X_{T}$ block matrices are the connection matrices between circuit nodes and contacts, and the MNA block matrix represents the modified nodal circuit analysis.

\section{RESULTS}

A rectangular connector $(1 \mathrm{~mm} \times 1 \mathrm{~mm} \times 4 \mathrm{~mm})$ is modeled to determine the convergence of the resistance curve around the first resonance of the structure. The results shown in Fig. 3 include the hybrid formulation (164 triangles and 216 tetrahedrons) and the boundary element method formulation (48 rectangles) from [5]. The complete basis order is denoted by $p$. The various results agree well except when $p=0$ for the boundary element method since convergence had not been obtained.

\section{SUMMARY}

A hybrid boundary element/finite element formulation is coupled with a technique for representing circuit-to-EM connections. Higher-order bases functions and elements 
may be used in the analysis. Results were presented to validate the hybrid formulation compared to the boundary element formulation.

\section{ACKNOWLEDGMENTS}

This work was partially supported by DARPA-MTO NeoCAD grant N66001-01-1-8920.

This work was performed under the auspices of the U.S. Department of Energy by University of California, Lawrence Livermore National Laboratory under Contract W7405-Eng-48.

\section{REFERENCES}

[1] R.M. Sharpe, J.B. Grant, N.J. Champagne, W.A. Johnson, R.E. Jorgenson, D.R. Wilton, W.J. Brown, and J.W. Rockway, "EIGER: Electromagnetic Interactions GEneRalized," 1997 IEEE AP-S International Symposium and North American URSI Radio Science Meeting, Montreal, Canada, July 1997, pp. 2366-2369.

[2] P.W. Fink and D.R. Wilton, "Higher order modeling in the BEM and hybrid BEM/FEM formulations," Ph.D. dissertation, Univ. Houston, Houston, TX, 2002.

[3] R.D. Graglia, D.R. Wilton and A.F. Peterson, "Higher order interpolatory vector bases for computational electromagnetics," IEEE Trans. Antennas and Propagation, vol. 45, No.3, pp. 329-342, Mar. 1997.

[4] V. Jandhyala and C. Yang, "A time domain surface integral technique for mixed electromagnetic and circuit simulation," Proc. IEEE meeting on Electric. Perf. of Electron. Packaging, San Jose, October 2002, pp. 41-44.

[5] N.J. Champagne, J.D. Rockway, and V. Jandhyala, "Mixed electromagnetic and circuit simulations using higher-order elements and bases," 2003 International Conference on Electromagnetics in Advanced Applications, Torino, Italy, September 2003, pp. 103-106.

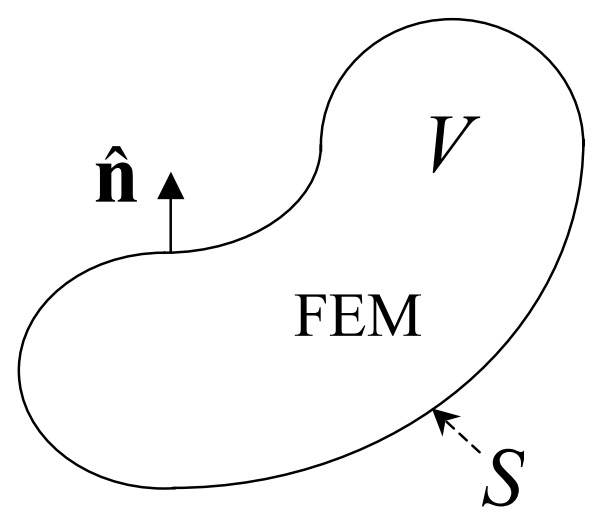

Figure 1. A body is in space where the volume $V$ is surrounded by the surface $S$. 


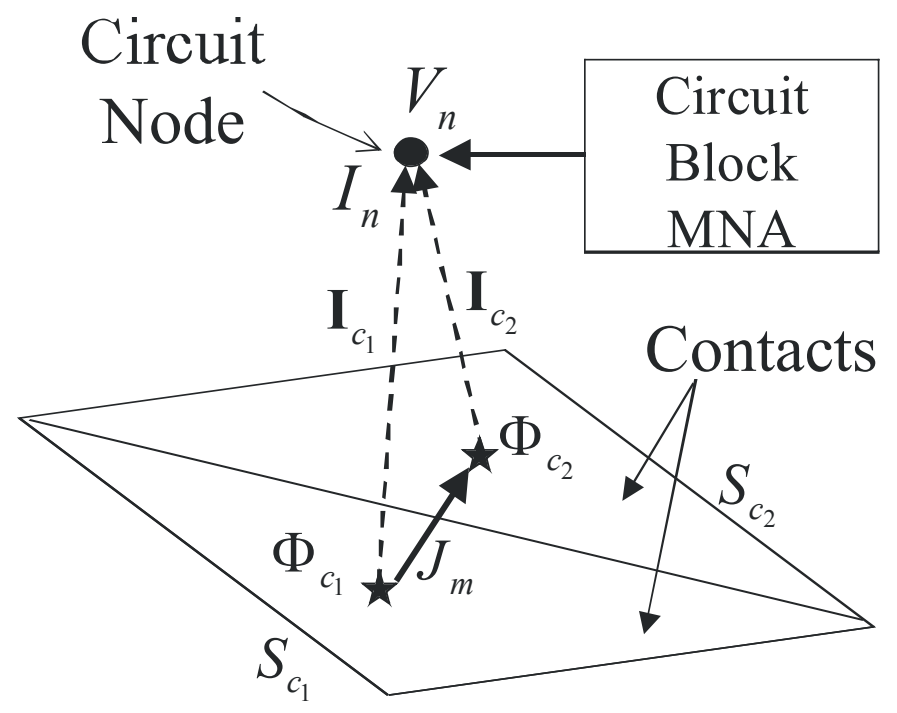

Figure 2. This schematic shows the approach for creating the contact-circuit connection.

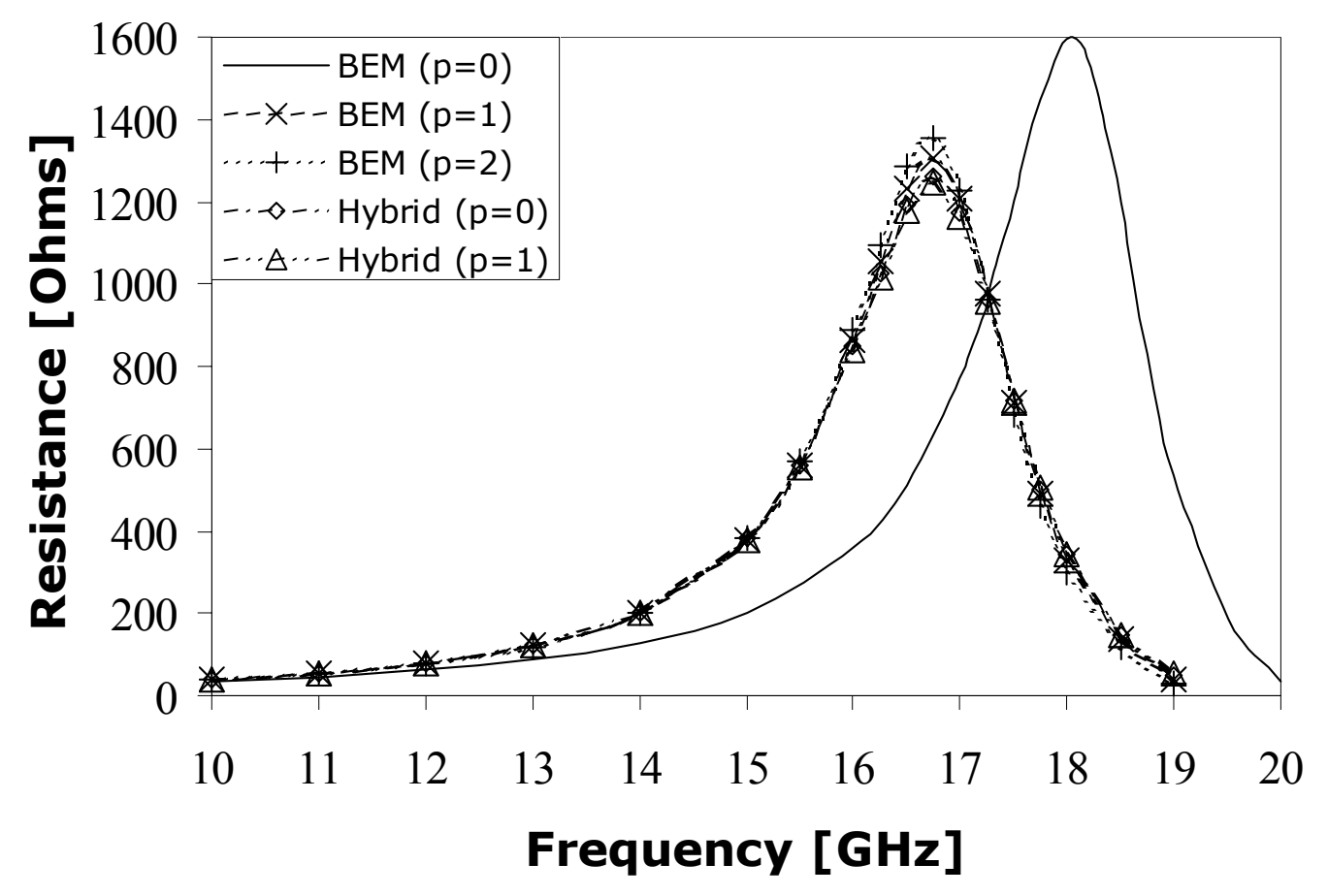

Figure 3. This is a plot of the resistance versus frequency for a rectangular connector. The connector was modeled using a boundary element method (BEM) and hybrid BEM/FEM formulation (FEM). The complete basis order $p$ for each case is shown in the legend. 